RESEARCH

\title{
Testing the effects of educational toilet posters: A novel way of reducing haemolysis of blood samples within ED
}

\author{
David Corkill, RN, MEmergN, MAdvPrac(HthProfEdu)*
}

\author{
Emergency Nurse Educator, Emergency Department, Gold Coast Hospital, Southport, 108 Nerang St, Southport, Qld 4215, \\ Australia
}

Received 3 August 2011; received in revised form 21 October 2011; accepted 14 November 2011

\section{KEYWORDS \\ Posters; \\ Toilet; \\ Education; \\ Educational \\ Research; \\ Haemolysis; \\ Blood specimen \\ collection; \\ Emergency}

\begin{abstract}
Summary Haemolysed blood samples are an unnecessary burden on Emergency Departments (ED) as they increase workloads and drive down efficiencies. Little empirical data exists that demonstrates the effectiveness of educational posters displayed in staff toilet cubicles. This study explored the impact educational toilet posters have on reducing haemolysis rates within the ED.

Methods: A time series study of the clinical effect of educational toilet posters on reducing haemolysis rates throughout a 12 month period at the Gold Coast Hospital ED was undertaken. The GCH ED is a tertiary emergency service that has approximately 66,000 patient presentations per year. Data was collected prospectively. Analysis was undertaken to investigate the effects on total number of haemolysed samples and those clinically significant samples with a haemolytic index $>3$. Further investigation explored the specific effects on medical and nursing staff.

Results: Analysis undertaken using an independent $t$-test found that the pre-intervention data demonstrates a medium haemolysis rate of $4.92 \%(S D=1.04)$. This is a statistically significantly different $(t=3.56, d f=50, p=0.001)$ from the median post intervention data of $3.95 \%$ $(S D=0.84)$. The difference of $0.97 \%(95 \% \mathrm{Cl}=0.42,1.52)$ represents a $19.72 \%$ reduction in clinically significant haemolysed samples over the study period.

Conclusion: This study reveals that the use of educational toilet posters had a positive impact on reducing the rates of haemolysed samples collected within the ED. This simple and cost effective educational initiative changed the behaviour of clinical staff. Further investigation is warranted to examine the impact of educational toilet posters on additional clinical scenarios. Crown Copyright @ 2011 Published by Elsevier Ltd on behalf of College of Emergency Nursing Australasia Ltd. All rights reserved.
\end{abstract}

\section{Introduction}

* Tel.: +61 755198407.

E-mail address: David_corkill@health.qld.gov.au
Haemolysis is the breakdown of red blood cells which leads to the leaking of intracellular contents into the plasma. 


\section{What is known}

- The causes of haemolysed blood samples within the Emergency Department have been well documented.

- However, there has been little research into strategies using this evidence to reduce haemolysis of blood samples in clinical practice in emergency departments.

- Poster education is frequently used as a mode of transmission of knowledge to clinical staff yet the effectiveness of this methodology has not been demonstrated within the Emergency Department.

\section{What this paper adds}

- Educational toilet posters were an effective way of decreasing the amount of haemolysed blood samples within a tertiary Emergency Department.

- Toilet posters can assist educators and other hospital staff to change clinical practice based on emerging evidence through an efficient an effective methodology.
However the evidence for positive effects on the clinician's behaviour in the literature is unclear.

The effectiveness of educational toilet posters is based on Knowles Andragogy Theory of Learning. ${ }^{16}$ Following this theory, participants learn best when an internal motivator is developed and the learners are provided with clear and practical outcomes. The toilet is an environment where there is little distraction and staff may readily absorb information provided. The assessment of the effectiveness of educational toilet posters is based around Kirkpatrick's Model of Training Evaluation. ${ }^{17}$ Within this model, the research is focusing, not on the individual, the trainer or the educational program, but on the result of the educational intervention.

The research question this study explored was: Does the use of educational toilet posters have an impact the outcomes of clinical care? The aim of the study was to investigate the effectiveness of educational posters placed in staff toilet cubicles on the rates of haemolysed blood samples collected from patients attending an ED. The study also examined the effect of educational toilet posters on specific groups within the multidisciplinary team, specifically nursing and medical teams.

\section{Methods}

Haemolysis can occur during the process of collecting blood samples. If significant haemolysis has occurred then this may render the blood sample unusable. ${ }^{1}$ This has a significant impact on the efficient running of both the emergency and pathology departments as well as a negative effect on patients and the care they receive whilst within the ED. ${ }^{2}$

A number of authors have examined factors that effect haemolysis. ${ }^{1-8}$ However, there is a paucity of articles that have investigated strategies that influences change in clinical practice in relation to haemolysis. One study used a surveillance strategy within the ED with limited response. ${ }^{9}$ Another study successfully investigated strategies in reducing haemolysis rates within an ED. ${ }^{10}$ Although the authors were successful in reducing the rates of haemolysis within their ED, the effort required for the education intervention was considerable. The education used was predominately inservice based and in order to maintain its effectiveness, educational inservice's were repeated every six months to accommodate for staff turnover.

A small number of articles have investigated the use of educational posters within the clinical area which have demonstrated mix results. ${ }^{11-13}$ In a systematic review of the literature, only two articles discuss the use of educational toilet posters. One article provided an overview of their positive use within their department, however the authors were unable to provide a research design or statistical evidence of their effectiveness. ${ }^{14}$ The second study demonstrated a statistically significant change in the knowledge of CPR that was directly linked to educational toilet posters. ${ }^{15}$ However, this study explored the effects of educational toilet posters on undergraduate nursing students living in dormitory accommodation rather than a clinical environment.

Providing ongoing education to large groups of individuals within an ED remains a challenge. Anecdotal evidence suggests that there is a positive effect on the clinician's behaviour through the use of educational toilet posters.
This study employed a quasi-experimental design utilising a time series approach. Data was collected prospectively during the study period of twelve months. Data was collected weekly to decrease the variability in samples from week to week. At the completion of the first eight months data was examined to identify the pre intervention rates of haemolysis. This timeframe also ensured that any education on haemolysis before undertaking the study would not have an impact on the studies results. Data collection continued for a further four months following the placement of the educational toilet posters.

The dependent variable was the rate of haemolysis. This data were collected automatically through routine pathology recording software. All blood samples sent to pathology for biochemical analysis from the ED were included. The biochemical analysis was undertaken using a Beckman Unicel DxC600i analyser which includes a ranking system using the Haemolytic Index $(\mathrm{HI})$. This graded the sample from 0 to $10+$. All samples graded with a $\mathrm{HI}>3$ are automatically rejected by the analyser as it is unable to analyse these samples accurately. Samples that have an $\mathrm{HI}>3$ are then recorded electronically as a 'no test'. At no time was the investigator responsible for the $\mathrm{HI}$ analysis.

Blood samples with an $\mathrm{HI}>3$ then underwent further analysis to identify the status of the blood sample collector (medical, nursing or unknown). Each pathology sample was accompanied by a pathology request from which was electronically attached to the blood samples results. Each haemolysed sample was reviewed by a research assistant to determine the clinical status of the staff member who collected the blood. The investigator reviewed $10 \%$ of the samples to ensure accuracy of data. The investigator also reviewed any cases of 'Unknown' collector identification to determine if a decision could be made regarding the exact identification of the sample collector. 
The independent variable is a laminated educational poster (see Fig. 1) that was placed within each staff toilet cubicle within the ED. Throughout the intervention period, regular surveillance of the posters was undertaken to ensure the posters had not been inadvertently removed. The post intervention data collection started one week after the educational toilet posters were placed in the staff cubicles.

The educational poster displayed a number of different components aimed at providing information as well as educational content. The posters were colour printed to an A3 format. As well as providing information on current haemolysis rates, posters also provide strategies to reduce haemolysis based on evidence. The key points were

- Avoid taking blood through an intravenous cannula ${ }^{1,5-8}$

- Avoid using vacutainers to collect blood ${ }^{5,10}$

- Avoid collecting blood from difficult cannulation ${ }^{2,4}$

- If blood has to be taken from a cannula, use at least an 18 gauge $^{2,8}$

- When undertaking venipuncture, the antecubitial vein is the preferred site $^{1-3,6}$

A number of factors may have had effect on the dependent variable which is the rate of haemolysed blood samples collected within the ED. This may include variance from the usual pathology collection equipment, intravenous cannula or pathology collection devices. Pathology processing or analysis could also have had an effect on the dependent variable. However, it was determined that there was no change in practice of blood collection or pathology processing that was initiated throughout the study period.

As any further educational processes on haemolysis would be considered a variable and therefore impact on the dependent variable, all education on ways of reducing haemolysis were postponed during the study period. However if clinical staff started to discuss haemolysis once the posters were released, there discussions were not discouraged as the posters had the desired effect of raising awareness of haemolysis. The investigator did not instigate conversations around haemolysis throughout the study period.

Data was analysed for normal distribution using skewness and kurtosis for the continuous data. These are summarised as mean and standard deviation. Due to the large variation in the number of weekly blood samples collected, the weekly percentage of haemolysis was utilised for assessment. Comparisons were made using independent $t$-tests. A $p$ value of $<0.05$ was considered statistically significant. A Levene's test of equality of variance was used to test for variance whilst undertaking the $t$-test. If $p=<0.05$ then there is not equal variance between the groups. Statistical analysis was performed using the SPSS v.19 statistical software (SPSS, Chicago, IL, USA).

Ethics approval was granted from the Human Research Ethics Committee of Gold Coast Health Service District.

\section{Results}

All continuous data was found to be parametric in distribution. An independent sample $t$-test was conducted to evaluate if there was a difference between the pre and post intervention percentage rates of blood samples with a $\mathrm{HI}>3$.
The pre intervention rates $(M=4.93, S D=1.05)$ is statistically significantly different $(t=3.56, d f=50, p=0.001)$ from that of post intervention rates $(M=3.95, S D=0.84)$. The mean difference between pre and post intervention rates of blood samples with a $\mathrm{HI}>3$ was $0.97(95 \% \mathrm{Cl}=0.42,1.52)$. Because the variances for the two groups were significantly equal $(F=1.18, p=0.282)$ a test for equal variances was used.

The study also analysed the rates of any blood samples which detected any level of haemolysis. It was found that the mean score for pre intervention rates was $(M=55.04$, $\mathrm{SD}=3.27)$ also statistically significantly different $(t=8.35$, $d f=50, \quad p=0.000$ ) from that of post intervention rates $(M=48.13, S D=2.31)$. The mean difference of samples that contained any level of haemolysis was $6.91(95 \% \mathrm{Cl}=5.24$, $8.57)$. Because the variances for the two groups were significantly equal $(F=3.93, p=0.053)$ a test for equal variances was used.

The difference in rates of blood samples with a $\mathrm{HI}>3$ that was either collected by nursing, medical or unknown staff members was also assessed using an independent $t$-test. This found there was a significant statistical difference $(t=3.95$, $d f=50, p=.000)$ in the pre intervention percentage of samples collected by nursing staff $(M=2.54, S D=.92)$ compared to the post intervention group $(M=1.64, S D=.61)$. The mean difference of samples with a $\mathrm{HI}>3$ that was collected by nursing staff was $0.90(95 \% \mathrm{Cl}=.44,1.36)$. The variances for the two groups were significantly equal $(F=1.62, p=.208)$ therefore a test for equal variances was used.

However the differences between the pre and post intervention groups of blood samples with a $\mathrm{HI}>3$ that were collected by medical staff did not reach statistical significance $(t=.71, d f=50, p=.483)$. Similarly there was no statistical significant difference between the pre and post intervention group of blood samples with a $\mathrm{HI}>3$ for the unknown collector $(t=-1.38, d f=50, p=.173)$.

\section{Discussion}

This study demonstrates the use of educational toilet posters does reduce the rate of haemolysis in those samples with a $\mathrm{HI}>3$. The reduction in this group has implications for the care provided to those patients within the ED including reduced length of stay, reduced numbers of procedures and reduced costs to the ED. The decrease in $\mathrm{HI}>3$ blood samples represents a $19.7 \%$ reduction overall. However, according to the American Society of Clinical Pathology ${ }^{7}$ a $2 \%$ haemolysis rate for ED is considered best practice. Therefore, the reduction may actually represent a $33.1 \%$ improvement in samples with a $\mathrm{HI}>3$.

There was also a significant improvement in the overall haemolysis levels detected amongst blood samples. The mean reduction of 6.91 represents a $12.5 \%$ change in the pre and post intervention results. Any level of haemolysis means potential errors in biochemistry results, therefore this reduction is clinically significant. Not only does the reduction in the amounts of haemolysed blood samples improve the accuracy of the result, it also decreases the time spent by pathology staff correcting results for haemolysed blood samples.

The majority of blood samples with a decreased $\mathrm{HI}>3$ occurred within the nursing staff group. Although there was 
Table 1 Results of intervention on percentage of haemolysed samples.

\begin{tabular}{|c|c|c|c|c|c|}
\hline \multirow[t]{2}{*}{ Variable } & \multicolumn{2}{|c|}{$\begin{array}{l}\text { Haemolysed samples percentage of total } \\
\text { test }\end{array}$} & \multirow{2}{*}{$\begin{array}{l}\text { Percentage } \\
\text { difference, } \mathrm{M} \\
(95 \% \mathrm{Cl})\end{array}$} & \multirow[t]{2}{*}{$t$} & \multirow[t]{2}{*}{$p$ value } \\
\hline & $\begin{array}{l}\text { Pre intervention, } \\
M(S D)\end{array}$ & $\begin{array}{l}\text { Post intervention, } \\
M(S D)\end{array}$ & & & \\
\hline Samples with $\mathrm{HI}^{\mathrm{a}}>3$ & $4.93(1.05)$ & $3.95(0.84)$ & $0.97(0.42,1.52)$ & 3.557 & 0.001 \\
\hline Any Haemolysis & $55.04(3.27)$ & $48.13(2.31)$ & $6.91(5.24,8.57)$ & 8.35 & 0.000 \\
\hline $\mathrm{HI}^{\mathrm{a}}>3$ collected by Nurse & $2.54(0.92)$ & $1.64(0.61)$ & $0.90(0.44,1.36)$ & 3.95 & 0.000 \\
\hline $\mathrm{HI}^{\mathrm{a}}>3$ collected by Doctor & $2.27(0.59)$ & $2.14(0.73)$ & $0.13(-0.24, .50)$ & 0.71 & 0.483 \\
\hline $\mathrm{HI}^{\mathrm{a}}>3$ collected by Unknown & $0.12(0.14)$ & $0.17(0.16)$ & $0.06(-0.14,0.03)$ & -1.38 & 0.173 \\
\hline
\end{tabular}

a Haemolytic index ( $>3$ is clinically significant).

a reduction in the percentage of samples that were collected by medical staff, this was not statistically significant. It is unclear why the effect of the educational toilet poster was greater in nursing staff than the medical staff. This study did not set out to explore the pre-existing knowledge of haemolysis or the motivators for change in relation to poster education. However, these issues would be a worthwhile topic for further research.

This study examined pathology collection between the multidisciplinary groups of nursing and medical staff. The study was unable to determine if particular individuals were collecting more haemolysed samples than their colleagues. However, if individuals could have been identified, education and individual counselling could be provided to investigate strategies that could further reduce the amount of blood samples with a $\mathrm{HI}>3$.

Developing education posters is not a simple process. To develop a quality poster with clear messages that provides an educational impact takes considerable time. Many educational posters seen in the clinical environment lack simple design impact. The poster is often lost in the sea of posters and information overload that often inundates the clinical workspace.

Using educational posters in staff toilet cubicles does have an impact on the clinical environment. This is an environment were staff have downtime from the hectic workload within the ED to be able to absorb knowledge. Providing education to large groups of staff remains a challenge to emergency nurse educators. The use of educational toilet posters provides an efficient way of delivering ongoing education to large groups.

Whilst it is worthwhile to utilise this space to provide an educational opportunity, careful selection of the educational message and presentation style needs to be undertaken to ensure transfer of knowledge into change within the clinical environment. There is also a risk that this teaching space could become over saturated with educational posters and staff becoming immune to the information provided.

\section{Limitations}

One of the major limitations of the study is the design process. This time series design study does not have a control group or randomisation of subjects therefore making it difficult to provide categorical connections between the intervention and the outcome (Table 1).

Due to the design of the study, there could be extraneous effects the author is unaware of that may have had an influence on the outcomes of the study. During the study period participants may have been involved in education on haemolysis the researcher was unaware of or a specimen handling process undertaken outside of the ED that would impact on haemolysis rates.

This study was not accessing the experience levels of the blood sample collectors. If that level of detail was collected, this may have shown that throughout the study the inexperienced staff became more skilled and therefore reduce the amount of haemolysed samples. However, the GCH ED has a stable workforce and the inexperienced practitioner undertakes only a small proportion of blood samples collected within the ED. Due to the study design, changing experience level throughout the study period cannot be categorically ruled out as rationale for change in haemolysis rates.

\section{Provenance and conflict of interest}

The author has no potentially conflicting interests to declare. This paper was not commissioned.

\section{Funding interests}

No funding sources require acknowledgement.

\section{Acknowledgements}

The author wishes to acknowledge and thank Christine Heenan, Rebecca Saunders and Christina Betts for their assistance with data collection during the study as well Alison Willsher for manuscript editing.

\section{Appendix A.}

See Fig. 1. 


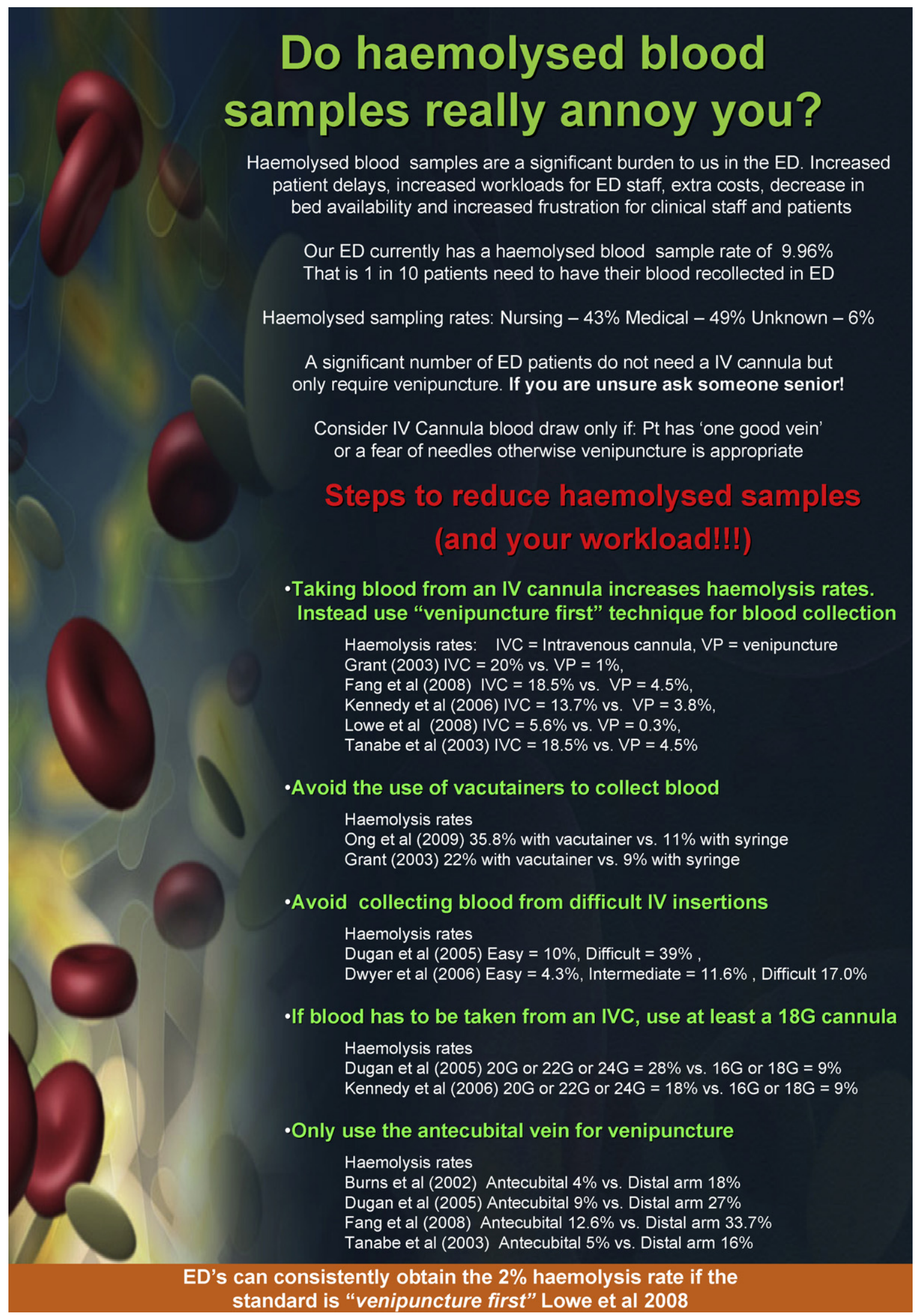

Figure 1 Poster used for Toilet Education. 


\section{References}

1. Fang L, Fang S, Chung $\mathrm{Y}$, Chien S. Collecting factors related to the haemolysis of blood specimens. Journal of Clinical Nursing 2008;17(17):2343-51.

2. Dugan L, Leech L, Speroni K, Corriher J. Factors affecting hemolysis rates in blood samples drawn from newly placed iv sites in the emergency department. Journal of Emergency Nursing 2005;31(4):338-45.

3. Burns E, Yoshikawa N. Hemolysis in serum samples drawn by emergency department personnel versus laboratory phlebotomists. Laboratory Medicine 2002;33(5):378-80.

4. Dwyer D, Fry M, Somerville A, Holdgate A. Randomized, single blinded control trial comparing haemolysis rate between two cannula aspiration techniques. Emergency Medicine Australasia 2006;18(5):484-8.

5. Grant $M$. The effect of blood drawing techniques and equipment on the hemolysis of ED laboratory blood samples. Journal of Emergency Nursing 2003;29(2):116-21.

6. Tanabe P, Kyriacou D, Garland F. Factors affecting the risk of blood bank specimen hemolysis. Academic Emergency Medicine 2003;10(8):897-900.

7. Lowe G, Stike R, Pollack M, Bosley J, O’Brien P, Hake A, et al. Nursing blood specimen collection techniques and hemolysis rates in an emergency department: analysis of venipuncture versus intravenous catheter collection techniques. Journal of Emergency Nursing 2008;34(1):26-32.

8. Kennedy C, Angermuller S, King R, Noviello S, Walker J, Warden $\mathrm{J}$, et al. A comparison of hemolysis rates using intravenous catheters versus venipuncture tubes for obtaining blood samples. Journal of Emergency Nursing 1996;22(6): 566-9.

9. Pretlow L, Gandy T, Leibach EK, Russell B, Kraj B. A quality improvement cycle: hemolyzed specimens in the emergency department. Clinical Laboratory Science 2008;21(4):219-24.

10. Ong $M$, Chan $Y$, Lim C. Reducing blood sample hemolysis at a tertiary hospital emergency department. American Journal of Medicine 2009;122(11), 1054.e1-6.

11. Badland $H$, Schofield $G$. Posters in a sample of professional worksites have no effect on objectively measured physical activity. Health Promotion Journal of Australia 2005;16(1): 78-81.

12. Morse $L, M c D o n a l d ~ M$. Failure of a poster-based educational programme to improve compliance with peripheral venous catheter care in a tertiary hospital. A clinical audit. Journal of Hospital Infection 2009;72(3):221-6.

13. Qureshi A, Hughes N, Murphy E, Primrose W. Factors influencing uptake of influenza vaccination among hospital-based health care workers. Occupational Medicine 2004;54(3):197-201.

14. Simmons P, Launius B. Bathroom blitz it! The Journal of Continuing Education in Nursing 1997;28(5):235-7.

15. Grogono A, Johnson M, Jastremski M, Russell R. Educational graffiti: better use of the lavatory wall. The Lancet 1982; 319(8282):1175-6.

16. Knowles M. The adult learner: a neglected species. Houston: Gulf Publishing; 1973.

17. Kirkpatrick D. Techniques for evaluating training programs. Alexandria: ASTD; 1975. 